\title{
Proton magnetic resonance spectroscopic imaging in the clinical evaluation of patients with Niemann-Pick type $\mathrm{C}$ disease
}

G Tedeschi, S Bonavita, N W Barton, A Bertolino, J A Frank, N J Patronas, J R Alger, R Schiffmann

Neuroimaging Branch G Tedeschi S Bonavita

Developmental and Metabolic Neurology Branch, NINDS

$\mathrm{N}$ W Barton

R Schiffmann

Clinical Brain Disorders Branch, NIMH

A Bertolino

Laboratory of Diagnostic Radiology Research

J A Frank

Department of

Diagnostic Radiology, National Institutes of Health, Bethesda, MD, USA

N J Patronas

Department of Radiological Sciences, University of California, Los Angeles, CA, USA J R Alger

Correspondence to: Professor Gioacchino Tedeschi, Istituto di Scienze Neurologiche, Seconda Universita' di Napoli, Via Pansini 5, 80131 Napoli, Italy. Telephone 003981566 6786; fax 0039815666787 .

Received 28 May 1997 and in revised form 30 September 1997

Accepted 4 December 1997

\begin{abstract}
Objectives-10 patients with NiemannPick disease type C (NP-C) were studied by proton magnetic resonance spectroscopic imaging ( ${ }^{1} \mathrm{H}$-MRSI) to assess the biochemical pathology of the brain and to determine whether this method can be useful to clinically evaluate these patients. Methods- ${ }^{1}$ H-MRSI permits the simultaneous measurement of $\mathrm{N}$-acetyl aspartate (NA), compounds containing choline (Cho), creatine plus phosphocreatine (Cre), and lactate (Lac) signal intensities from four $15 \mathrm{~mm}$ slices divided into 0.84 ml single volume elements. Spectroscopic voxels were identified from seven regions of interest.

Results-In patients with NP-C, NA/Cre was significantly decreased in the frontal and parietal cortices, centrum semiovale, and caudate nucleus; Cho/Cre was significantly increased in the frontal cortex and centrum semiovale. Significant correlations were found between clinical staging scale scores and ${ }^{1} H$-MRSI abnormalities. Conclusion- ${ }^{1}$ H-MRSI showed diffuse brain involvement in patients with NP-C consistent with the pathological features of the disease. ${ }^{1} \mathrm{H}$-MRSI is an objective and sensitive tool to neurologically evaluate patients with NP-C.

(F Neurol Neurosurg Psychiatry 1998;65:72-79)
\end{abstract}

Keywords: proton magnetic resonance spectroscopy; Niemann-Pick; clinical stage

Niemann-Pick disease type C (NP-C) is an autosomal recessive lipidosis, characterised by a unique error in the intracellular trafficking of lysosomal cholesterol. ${ }^{1}$ This abnormality forms the basis for the biochemical diagnosis of the disease, which requires confirmation of both impaired cholesterol esterification by cultured skin fibroblasts and visualisation (by filipin staining) of excess accumulation of free cholesterol in their lysosomes. ${ }^{2}{ }^{3}$ The disease shows a broad clinical range with diverse neurological manifestations including psychomotor deterioration, ataxia, vertical supranuclear palsy, and developmental delay. ${ }^{4}$ Conversely, pathological and histological abnormalities are more unifying features, with the presence of storage material in ballooned neurons in the brain. Ultrastructurally, the storage material occurs as membranous and osmiophilic granules in lysosomes. Subse- quently there are destructive and degenerative abnormalities with widespread neuronal loss, and to a minor extent, astrogliosis and demyelination. $^{56}$

Niemann-Pick type C disease is a diffuse, degenerative brain disease, and although a clinical staging scale has recently been published, ${ }^{4}$ it has a relatively limited sensitivity as a measure of the degree of brain involvement. Thus there is a need for a technique that could accurately assess the amount of brain involvement.

Proton magnetic resonance spectroscopic imaging ( $\left.{ }^{1} \mathrm{H}-\mathrm{MRSI}\right)$ provides a non-invasive method to obtain information on brain metabolism, and has been successfully applied to the study of CNS disorders. ${ }^{7-12}$ The principal metabolite signals detected by ${ }^{1} \mathrm{H}$-MRSI at long echo time (TE) are compounds containing the $\mathrm{N}$-acetyl group with $\mathrm{N}$-acetyl aspartate (NA) as the prominent contributor, compounds containing choline (Cho), creatine plus phosphocreatine (Cre), and lactate (Lac). The multislice ${ }^{1} \mathrm{H}-\mathrm{MRSI}$ technique used in the present study permits simultaneous acquisition of NA, Cho, Cre, and Lac signal intensities from four brain sections, ${ }^{13}$ and enables spatial mapping of metabolic abnormalities.

In the present study of patients with NP-C, our goals were to determine whether multislice ${ }^{1} \mathrm{H}-\mathrm{MRSI}$ could (a) detect specific cortical and subcortical neuronal loss or damage, and regionally specific chemical changes associated with astrogliosis and demyelination, and $(b)$ to correlate ${ }^{1} \mathrm{H}$-MRSI findings with the degree of clinical impairment.

Subjects and methods

We studied 10 patients with NP-C, six males and four females, aged 6 to 39 years, and 15 healthy volunteers as controls (15 to 40 years old).

PATIENT CHARACTERISTICS AND CLINICAL RATING SCALE

Table 1 shows the clinical and MRI characteristics of the patients. A clinical stage was assigned to each patient based on both a previously published clinical staging classification for NP-C 4 and on the clinical characteristics of each patient at the time of the ${ }^{1} \mathrm{H}$-MRSI. The clinical stages scale has five stages ranging from stage 0 , with only biochemical evidence of NP-C and no neurological abnormality, to stage 4 , which is the end stage of neurological involvement. 
Table 1 Clinical data on patients with NP-C in the study

\begin{tabular}{|c|c|c|c|c|}
\hline \multirow[b]{2}{*}{ Patient/sex } & \multirow{2}{*}{$\begin{array}{l}\text { Age studied } \\
\text { (y) lonset of } \\
\text { neurological } \\
\text { disease }\end{array}$} & \multirow[b]{2}{*}{ Clinical findings/clinical stage ${ }^{4}$} & \multicolumn{2}{|l|}{ MRI findings } \\
\hline & & & Brain atrophy & White matter hyperintensity \\
\hline $1 / \mathrm{F}$ & $10 / 8$ & VSNGP visual spatial perception abnormality, $\mathrm{A} / 1$ & Absent & Absent \\
\hline $2 / \mathrm{F}$ & $17 / \mathrm{NR}$ & Slow downgaze saccades, $\mathrm{A} / 0$ & Absent & Absent \\
\hline $3 / \mathrm{M}$ & $6 / 1$ & VSNGP, spasticity weakness, ataxia, dysarthria, IQ:53, AWA/3 & Absent & Mild \\
\hline $4 / \mathrm{M}$ & $32 / 15$ & VSNGP, speech apraxia, ataxia, dystonia, AWA $/ 3$ & Moderate cerebral and cerebellar & Marked \\
\hline $5 / \mathrm{M}$ & $22 / 8$ & $\begin{array}{l}\text { Severe dystonia, anarthria, dysphagia, A with support and } \\
\text { mild-to-moderate dementia/3 }\end{array}$ & Mild cerebral and cerebellar & Mild \\
\hline $6 / M$ & $39 / 18$ & $\begin{array}{l}\text { VSNGP, dysarthria, ataxia, mild dystonia, A with support IQ:68, } \\
\text { language, visuomotor deficit/3 }\end{array}$ & Mild cerebral & Absent \\
\hline $7 / \mathrm{M}$ & $20 / 6$ & $\begin{array}{l}\text { VSNGP, moderate dystonia, paranoid delusions, } \mathrm{A} \text {, mild to } \\
\text { moderate dementia/2 }\end{array}$ & Mild cerebral & Mild \\
\hline $8 / \mathrm{M}$ & $25 / 18$ & $\begin{array}{l}\text { VSNGP, dystonia, dysarthria, dysphagia, ataxia, AWA, IQ: } 65 \text {, } \\
\text { deficient language and visuomotor function } / 3\end{array}$ & Moderate cerebellar & Absent \\
\hline $9 / \mathrm{F}$ & $20 / 11$ & VSNGP, mild dysarthria, perceptual skills)/2 & Absent & Absent \\
\hline $10 / \mathrm{F}$ & $15 / 6$ & Complete quadriparesis, opthalmoparesis responsiveness, NA/4 & Moderate & Marked \\
\hline
\end{tabular}

$\mathrm{A}=$ ambulatory; $\mathrm{NA}=$ non-ambulatory; $\mathrm{AWA}=$ ambulatory with assistance; $\mathrm{NR}=$ not relevant; $\mathrm{VSNGP}=$ vertical supranuclear gaze palsy.

MRI STUDIES

Brain MRI of patients with NP-C were obtained, before the acquisition of the ${ }^{1} \mathrm{H}$ MRSI data, with a 1.5 Tesla magnet (GE Medical Systems, Milwaukee, WI, USA) with T1 (TR: 416-500 ms; TE 9-16 ms) and T2 weighted (TR 2000-4000 ms; TE 90-108 ms) pulse sequences. The acquired images were reviewed by an experienced neuroradiologist (NJP) in a blind fashion without previous knowledge of the ${ }^{1} \mathrm{H}-\mathrm{MRSI}$ and clinical findings. Two variables were examined: the incidence of brain atrophy and the presence and severity of white matter abnormalities.

To assess the presence of atrophy, the bifrontal diameter of the lateral ventricles was measured and compared against the transverse diameter of the skull at the same level as previously described. ${ }^{14}$ From these measurements a bifrontal ratio was obtained; a value $>33 \%$ was considered abnormal. ${ }^{14}$ With subjective criteria, the size of the cortical sulci in the cerebral hemispheres and in the cerebellum was also evaluated.

Abnormalities in the white matter were assessed by the presence of abnormal areas of increased signal intensity with the T2 weighted technique. A three grade scale (mild, moderate, or marked) was used to rate brain atrophy and white matter abnormalities.

${ }^{1} \mathrm{H}-\mathrm{MRSI}$ STUDIES

The ${ }^{1} \mathrm{H}-\mathrm{MRSI}$ studies were performed on the same 1.5 Tesla MR imager equipped with self shielded gradients with a previously described data acquisition procedure and a standard quadrature imaging head coil. ${ }^{13}$ Images were obtained at an angle of about $+20^{\circ}$ from the orbitomeatal line to depict the maximum number of spectroscopic voxels throughout the brain. Phase encoding procedures were used to obtain a $32 \times 32$ array of spectra from voxels with a nominal volume of $0.84 \mathrm{ml}(7.5 \mathrm{~mm} \times 7.5$ $\mathrm{mm} \times 15 \mathrm{~mm}$ ) within the selected slices. The ${ }^{1} \mathrm{H}-\mathrm{MRSI}$ data acquisition comprised a multiple slice spin echo slice selection with a repetition time (TR) of $2200 \mathrm{~ms}$ and a TE of $272 \mathrm{~ms}$. Outer volume signal saturation was used to suppress signals arising from the skull marrow and surface tissues. Four $15 \mathrm{~mm}$ thick slices with $2.5 \mathrm{~mm}$ interslice spacing were acquired. One patient (10) was studied with an earlier
${ }^{1} \mathrm{H}-\mathrm{MRSI}$ technique that allowed acquisition of a single $15 \mathrm{~mm}$ spectroscopic slice, which we located at the level of the centrum semiovale.

The raw data from each slice consisted of a set of 804 phase encoded full spin echoes. The phase encodings sample a circular region of the $\mathrm{k}$ space centred at the origin. Each echo consists of 256 complex points sampled $1 \mathrm{~ms}$ apart (sweep width $1 \mathrm{kHz}$ ). The echoes were apodised by multiplying the nth point by the factor:

$\sin (\mathrm{n} \pi / 128)$ for $\mathrm{n}<64$

1 for $64 \leqslant n \leqslant 192$

$\sin ((256-n) \pi / 128)$ for $n>192$

The echoes were zero filled to 512 points and Fourier transformed to the frequency domain; the $\mathrm{k}$ space at each point in the frequency domain was zero filled to form a $32 \times 32$ square matrix (with $\mathrm{k}=0$ at the centre), and the $\mathrm{k}$ space domain was apodised by multiplying spectrum $1, \mathrm{~m}(-15<1, \mathrm{~m}<16)$ by $\sin ((1-0.5) / 33) \sin ((\mathrm{m}-$ $0.5) / 33$ ) before Fourier transforming to yield a $32 \times 32$ array of spectra.

To generate metabolite signal intensity images, the following procedure was used: (a) the magnitude for each spectrum was computed; $(b)$ an automated peak picking program was used to identify the Cho, Cre, NA, and Lac peaks; (c) the peak identification was reviewed and manually changed when the automatic procedure had failed; and (d) spectra from voxels contaminated by fat were zeroed out. After the peaks in all the spectra were properly identified, metabolite values were assessed for each pixel by summing the magnitude spectrum over a frequency band of width $6 \mathrm{~Hz}(0.1$ ppm) centred at each peak. Magnitude values were integrated to produce four $32 \times 32$ arrays showing spatial variation of the strength of each signal in each of the selected slices.

The $3 \mathrm{~mm} \mathrm{~T} 1$ and T2 weighted MRIs were used to identify the spectroscopic voxels encompassing defined neuroanatomical structures within the brain. Regions of interest (ROIs, fig 1), containing an integral number of $0.84 \mathrm{ml}$ spectroscopic voxels, were identified from the frontal cortex, temporal cortex, occipital cortex, parietal cortex, cingulate gyrus, centrum semiovale, thalamus, and caudate, according to the following criteria: $(a)$ the spectroscopic voxels should fit entirely within 


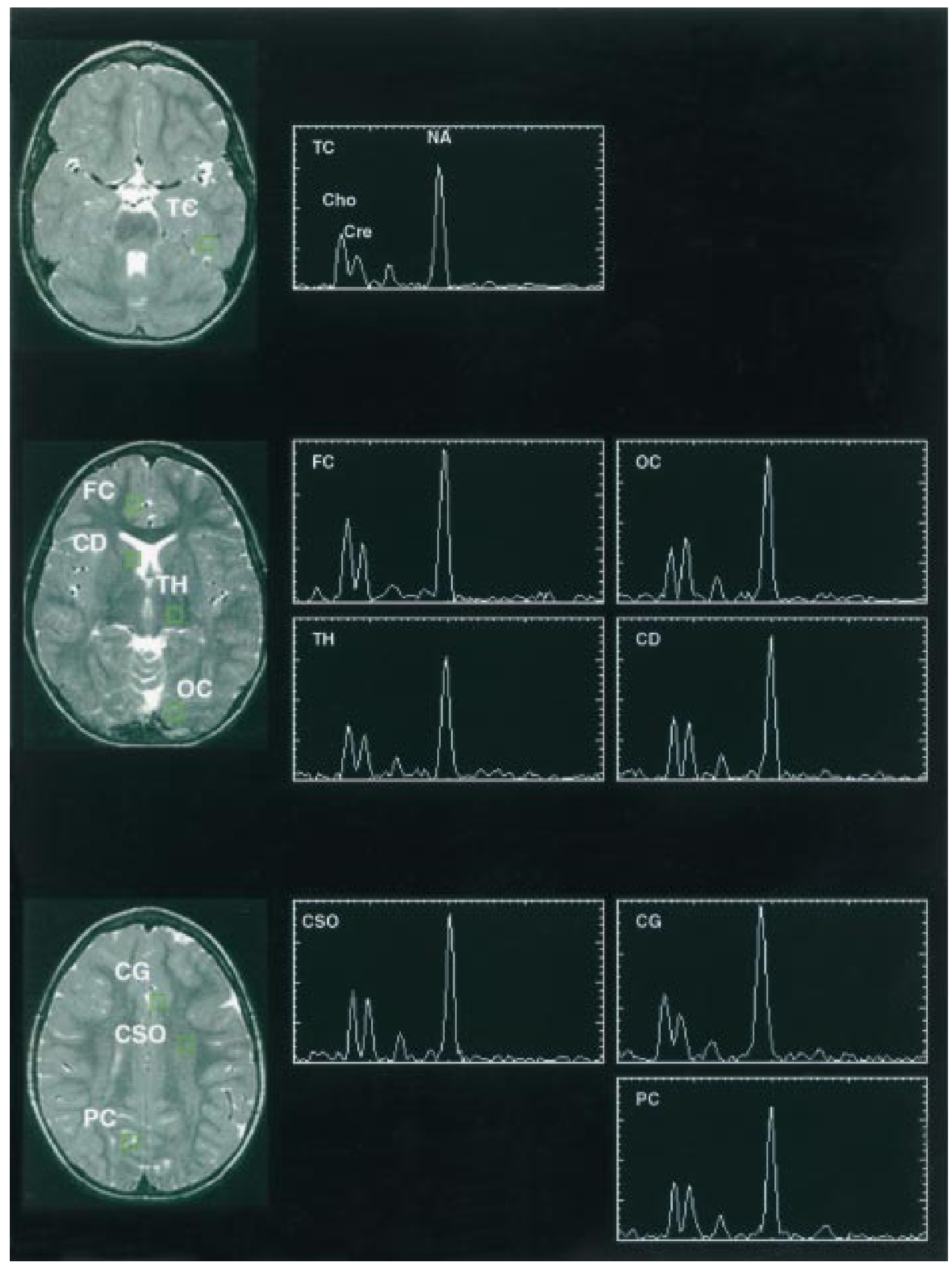

Figure 1 Location of the regions of interest (ROIs) and representative spectra in a control. The ROIs were selected from the frontal cortex (FC), temporal cortex (TC), occipital cortex (OC), parietal cortex (PC), cingulate gyrus (CG), centrum semiovale (CSO), thalamus (TH), and caudate (CD). Cho $=$ choline $;$ Cre $=$ creatine; $N A=N$-acetyl aspartate. 
Table 2 Abnormal ${ }^{1} H$-MRSI and clinical rating scale scores (CRSS) findings in patients with Niemann-Pick disease type C (NP-C) and controls

\begin{tabular}{|c|c|c|c|c|c|c|c|c|c|}
\hline$N P C$ & CRSS & $F C$ & $T C$ & $O C$ & $P C$ & $C G$ & CSO & $T H$ & $C D$ \\
\hline \multicolumn{10}{|l|}{ NA/Cre } \\
\hline 1 & 1 & $2.22 \downarrow$ & NS & NS & - & NS & $1.72 \downarrow$ & - & NS \\
\hline 2 & 0 & NS & - & NS & NS & - & NS & NS & NS \\
\hline 3 & 3 & $1.64 \downarrow$ & - & NS & - & - & NS & NS & $1.65 \downarrow$ \\
\hline 4 & 3 & $1.73 \downarrow$ & NS & NS & NS & - & $2.22 \downarrow$ & - & - \\
\hline 5 & 3 & $1.63 \downarrow$ & $1.83 \downarrow$ & - & NS & $1.50 \downarrow$ & $1.54 \downarrow$ & $2.03 \downarrow$ & $1.28 \downarrow$ \\
\hline 9 & 3 & $1.58 \downarrow$ & $1.85 \downarrow$ & - & - & NS & $1.96 \downarrow$ & - & - \\
\hline 7 & 2 & NS & NS & NS & NS & $1.63 \downarrow$ & $2.10 \downarrow$ & - & - \\
\hline 8 & 3 & $1.60 \downarrow$ & - & NS & - & - & NS & NS & NS \\
\hline 9 & 2 & $2.18 \downarrow$ & - & - & NS & - & NS & NS & - \\
\hline 10 & 4 & $1.31 \downarrow$ & - & - & - & - & $1.08 \downarrow$ & - & - \\
\hline Controls & $\begin{array}{l}\text { Mean (SD) } \\
\text { Range }\end{array}$ & $\begin{array}{l}2.83(0.30) \\
(2.55-3.70)\end{array}$ & $\begin{array}{l}2.65(0.17) \\
(2.20-4.40)\end{array}$ & $\begin{array}{l}2.59(0.43) \\
(1.50-3.10)\end{array}$ & $\begin{array}{l}2.52(0.25) \\
(1.65-2.84)\end{array}$ & $\begin{array}{l}2.39(0.21) \\
(2.11-3.79)\end{array}$ & $\begin{array}{l}2.81(0.26) \\
(2.58-4.12)\end{array}$ & $\begin{array}{l}2.54(0.22) \\
(1.98-3.31)\end{array}$ & $\begin{array}{l}2.53(0.23) \\
(1.68-2.87)\end{array}$ \\
\hline \multicolumn{10}{|l|}{ Cho/Cre } \\
\hline 1 & 1 & NS & NS & NS & - & NS & $1.87 \uparrow$ & - & NS \\
\hline 2 & 0 & NS & - & NS & NS & - & NS & NS & NS \\
\hline 3 & 3 & $1.80 \uparrow$ & - & NS & - & - & $2.00 \uparrow$ & NS & NS \\
\hline 4 & 3 & $1.79 \uparrow$ & NS & NS & NS & NS & NS & - & - \\
\hline 5 & 3 & $1.73 \uparrow$ & $1.60 \uparrow$ & - & NS & NS & $1.83 \uparrow$ & $1.75 \uparrow$ & NS \\
\hline 6 & 3 & $1.64 \uparrow$ & NS & - & - & NS & $1.99 \uparrow$ & - & - \\
\hline 7 & 2 & NS & NS & NS & NS & NS & NS & - & - \\
\hline 8 & 3 & $1.82 \uparrow$ & - & NS & - & - & $2.09 \uparrow$ & NS & - \\
\hline 9 & 2 & $1.64 \uparrow$ & - & - & NS & - & $2.28 \uparrow$ & NS & NS \\
\hline 10 & 4 & $2.15 \uparrow$ & - & - & - & - & $2.49 \uparrow$ & - & - \\
\hline \multirow[t]{2}{*}{ Controls } & Mean (SD) & $1.08(0.22)$ & $1.11(0.18)$ & $.95(0.12)$ & $1.20(0.24)$ & $1.09(0.17)$ & $1.24(0.20)$ & $1.24(0.20)$ & $1.32(0.30)$ \\
\hline & Range & $(0.86-1.50)$ & $(0.81-1.57)$ & $(0.61-1.29)$ & $(1.01-1.72)$ & $(0.82-1.81)$ & $(1.05-1.69)$ & $(1.06-1.67)$ & $(1.12-1.75)$ \\
\hline
\end{tabular}

For definition of abnormal ${ }^{1} \mathrm{H}-\mathrm{MRSI}$ values and abbreviations see methods.

- $=$ Not detected in ${ }^{1} \mathrm{H}-\mathrm{MRSI}$ data.

$\mathrm{FC}=$ frontal cortex; $\mathrm{TC}=$ temporal cortex $\mathrm{OC}=$ occipital cortex; $\mathrm{PC}=$ parietal cortex; $\mathrm{CG}=$ cingulate gyrus; $\mathrm{CSO}=$ centrum semiovale; $\mathrm{TH}=$ thalamus; $\mathrm{CD}=$ caudate.

the same neuroanatomical structure in at least four of the five $3 \mathrm{~mm}$ MRIs that corresponded to the $15 \mathrm{~mm}{ }^{1} \mathrm{H}-\mathrm{MRSI}$; and (b) voxels showing poor spectral resolution (less than half height separation of Cho and Cre signals) or residual water signal were excluded. The spectroscopic voxels were chosen by one of us (SB), who had no knowledge of the subject's clinical stage. The same inclusion criteria were also applied to the healthy controls. For each ROI, mean integrated metabolite signal intensity ratios (NA/Cre, Cho/Cre) from individual spectroscopic voxels were calculated for the patients and normal subjects. The number of spectroscopic voxels included in the ROIs was not predictive of the mean ratio values (as assessed by Spearman correlation analysis).

The intensities of metabolites from individual spectroscopic voxels within each ROI were averaged for comparison of group differences and tested by the Mann-Whitney $U$ test. The most abnormal metabolite ratios from individual spectroscopic voxels within each ROI were also separately assessed, and considered abnormal if they were smaller (NA/Cre), or larger (Cho/Cre) than the most abnormal value for a voxel within the corresponding ROI for each control (table 2). The degree of metabolic involvement was also assessed by taking into account the percentage of abnormal ROIs out of the total number of ROIs identified for each patient. Correlations between clinical staging, mean metabolite ratios, percentage of abnormal ROIs, brain atrophy, and white matter abnormalities were assessed by Spearman's correlation test.

\section{Results}

Figure 1 shows the location of the ROIs and the corresponding representative spectra in a patient. Figure 2 shows two sections of the ${ }^{1} \mathrm{H}-\mathrm{MRSI}$ and the corresponding MRI of one control and one patient with NP-C. In the present study, the images alone did not allow us to identify a pattern of regional abnormalities in any group of patients. The selected ${ }^{1} \mathrm{H}-$ MRSI sections are presented only to show the regional volume resolution of ${ }^{1} \mathrm{H}-\mathrm{MRSI}$ and are not representative of the behaviour of the whole group of patients.

Patients with NP-C showed a significant reduction of NA/Cre in the frontal cortex (1.87 (0.40), $\mathrm{p}<0.0003)$, parietal cortex $(2.16$ $(0.28), \quad \mathrm{p}<0.05)$, centrum semiovale $(2.09$ (0.64), $\mathrm{p}<0.01)$, and caudate (1.81 (0.41), $\mathrm{p}<0.03$ ), and a significant increase of Cho/Cre in the frontal cortex $(1.64(0.28), \mathrm{p}<0.001)$ and centrum semiovale (1.88 (0.37); $\mathrm{p}<0.001)$. Table 2 shows the control values (mean (SD), and range) for each ROI.

Table 2 shows the individual abnormal results of the ${ }^{1} \mathrm{H}-\mathrm{MRSI}$ examinations in patients with NP-C, as well as the clinical stage of each patient. The abnormal NA/Cre and Cho/Cre ratios are given for the spectroscopic voxels that fulfilled these inclusion criteria, whereas the empty cells represent nondetermined spectra (not fulfilling these criteria). Patients with NP-C showed a heterogeneous pattern of ${ }^{1} \mathrm{H}$-MRSI abnormalities. The $\mathrm{NA} / \mathrm{Cre}$ ratio was decreased in the frontal cortex (eight of 10 patients), temporal cortex (two of five patients), cingulate gyrus (two of four patients), centrum semiovale (six of 10 patients), thalamus (one of five patients), and caudate (two of five patients). The Cho/Cre ratio was increased in the frontal cortex (eight of 10 patients), temporal cortex (one of five patients), centrum semiovale (eight of 10 patients), and thalamus (one of five patients). No Lac signals were found in any ROI.

The involvement of individual patients was also assessed by the percentage of abnormal ROIs. Although this approach can be influenced by the number of selected good spectra (discussed already), we suggest that it may also 


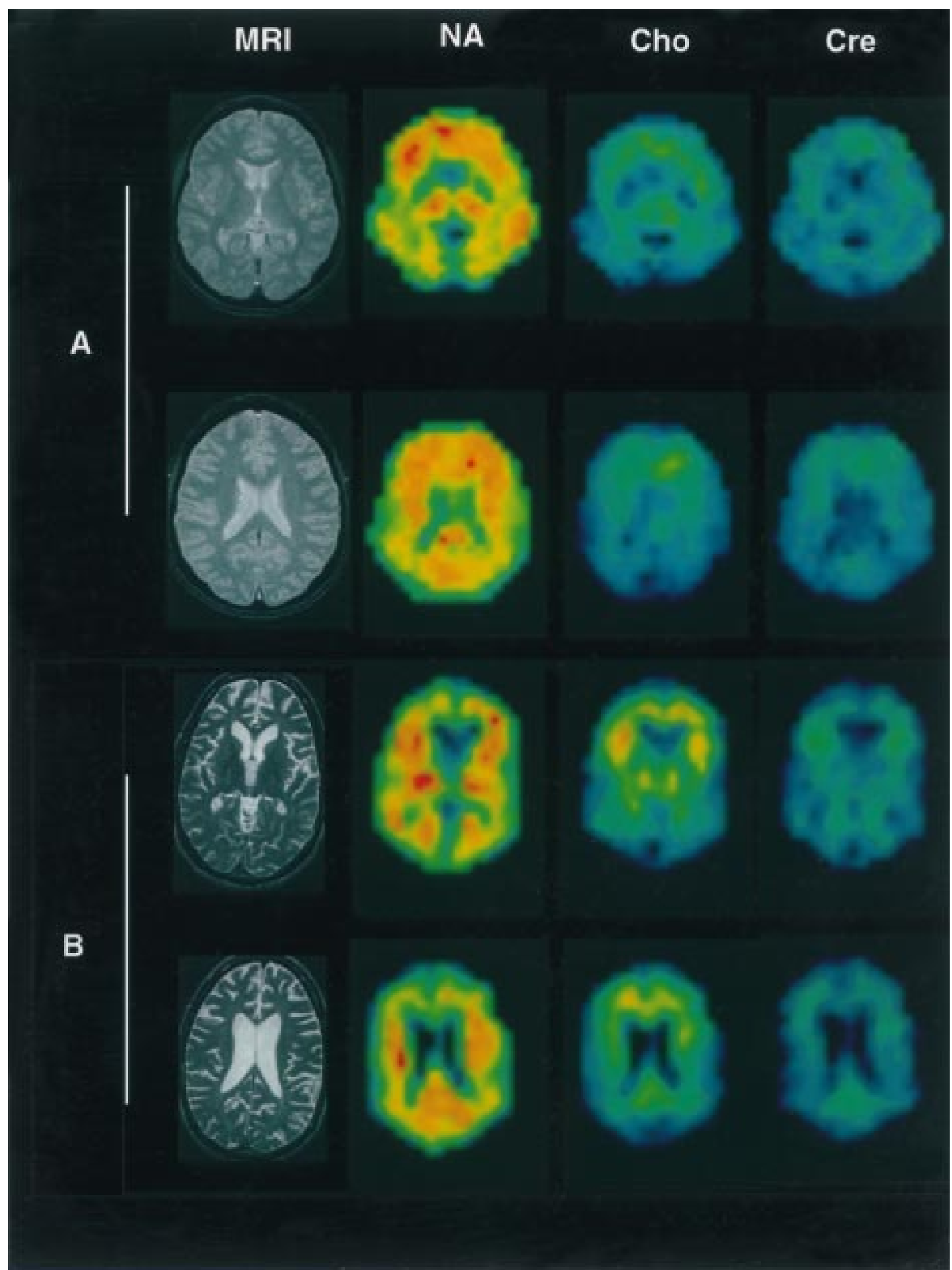

Figure 2 Two sections of the ${ }^{1} H-M R S I$ and corresponding MRI of $(A)$ a control and (B) a patient with NP-C (5). The MRI slice is 3 mm thick and corresponds to the centre of the $15 \mathrm{~mm}$ thick ${ }^{1} \mathrm{H}-\mathrm{MRSI}$ slice. Cho $=$ choline; $C r e=$ creatine; $N A=N$-acetyl aspartate. ${ }^{1} \mathrm{H}-\mathrm{MRSI}$ data are displayed with a colour scale which depicts the strongest signal integral with red and the weakest with dark blue. The ${ }^{1} H$-MRSI data are displayed at their nominal voxel in plane resolution $(7.5 \mathrm{~mm} \times 7.5 \mathrm{~mm})$. Colour images are scaled to the highest value of one the three metabolite signal intensities for each ${ }^{1} H-M R S I$, so that the pattern of regional distribution of metabolite signal intensities within the same slice can be compared between subjects, although colour intensity from the same anatomical location cannot be compared between subjects. 
represent a complementary way of determining severity of disease. The percentage of abnormal ROIs for both metabolite ratios ranged from $0 \%$ in the less affected patient (2) to $100 \%$ in the most affected patient (10). Note that patient 10 was studied by a previous single slice ${ }^{1} \mathrm{H}-\mathrm{MRSI}$, thus leading to a less comprehensive ROI selection (compared with the other nine patients). In two of the three patients with normal MRI, there were ${ }^{1} \mathrm{H}$-MRSI abnormalities.

Five of the 10 patients showed evidence of cerebral atrophy (table 1). In four of them, the lateral ventricles were enlarged and the cortical sulci were slightly widened, whereas in the fifth patient, who was less severely affected, brain atrophy was documented by ventricular enlargement. White matter abnormalities were found in five patients; four of them also had evidence of brain atrophy. In two of these patients, there was marked white matter involvement, whereas in the remaining three, involvement was mild. The periventricular and deep white matter structures were more severely affected with the subcortical regions being relatively spared. In four of the five patients $(4,5,7$, and 10) with white matter abnormalities, the centrum semiovale spectroscopic data partially included the affected white matter.

Significant correlations were found between clinical staging scale scores and NA/Cre in the frontal cortex $(r=-0.91 ; \mathrm{p}<0.0002)$ and caudate $(r=-0.97 ; \mathrm{p}<0.005)$ and Cho/Cre in the frontal cortex $(r=0.87 ; \mathrm{p}<0.001)$; percentage of abnormal ROIs for NA/Cre $(r=0.78 ; \mathrm{p}<.006)$, percentage of abnormal ROIs for Cho/Cre $(r=0.76 ; \mathrm{p}<0.01)$; and brain atrophy $(r=0.72$; $\mathrm{p}<0.02)$.

\section{Discussion}

The clinical range of NP-C is heterogeneous, and two clinical subgroups with five stages of severity within each group have been differentiated. ${ }^{4}$ Histological brain abnormalities include storage material in ballooned neurons and axons, axonal spheroids, neuronal and axonal loss, and to a minor extent, astroglyosis. $^{5}$ Neuronal ballooning is widespread, but is particularly evident in the basal ganglia, brain stem, and spinal cord. ${ }^{5}$ Neurofibrillary tangles, not associated with $\beta$-amyloid deposits, have been found in the cerebral cortex as well as in the basal ganglia, thalamus, and hypothalamus. ${ }^{6}$

$\mathrm{N}$-acetyl aspartate is inferred to be neuron specific because it is absent in both mature glial cell cultures and tumours of glial cell origin. ${ }^{15-19}$ Also, immunofluorescence studies have shown that fluorescent anti-NA antibodies colocalise with antibodies against neuron specific proteins..$^{20}{ }^{21}$ Recent experimental and clinical evidence suggests that NA may also be a marker of neuronal dysfunction. ${ }^{22-25}$ The present multislice ${ }^{1} \mathrm{H}$-MRSI study showed a diffuse pattern of neuronal involvement in patients with NP-C as indicated both by a significant reduction of NA/Cre (as group differences) in the frontal cortex, parietal cortex, centrum semiovale, and caudate and by the abnormal values in each patient. ${ }^{1} \mathrm{H}$-MRSI findings are consistent with the diffuse involvement of the CNS reported in previous neuropathological studies. ${ }^{5}$ In particular, the frequent presence of spectroscopic abnormalities in the frontal cortex in our patients parallels the predominant frontal lobe involvement that is found in postmortem analysis of NP-C brains. ${ }^{6}$ The topographical heterogeneity of our ${ }^{1} \mathrm{H}$-MRSI findings is also consistent with the diversified clinical presentation of patients with NP-C. ${ }^{4}$

The Cho peak reflects total brain choline stores, ${ }^{26}$ with major contributions from free choline, glycerophosphocholine, and phosphocholine. ${ }^{27}$ Increased Cho signal has been attributed to myelin breakdown as found in multiple sclerosis, ${ }^{28-30}$ some childhood white matter diseases, ${ }^{31-39}$ and increased turnover of membrane constituents, as found in brain tumours. ${ }^{40}{ }^{41}$ Higher concentrations of Cho are also found in astrocyte preparations. ${ }^{17}$ Therefore, we suggest that the significant Cho/Cre increase found in the frontal cortex and centrum semiovale of patients with NP-C may be regarded as an in vivo correlate of demyelination and astrogliosis.

Methods of obtaining absolute measures of signal intensity are not yet fully developed for ${ }^{1} \mathrm{H}$-MRSI techniques. Because additional time consuming scans are required, no effort was made in the present study to attain absolute signal levels. In such instances, the Cre signal is often used as a normalising factor to enable comparison between different brain regions and different subjects. The brain Cre MR signal has been studied in detail by Petroff et $a l .{ }^{42}$ The molecules (creatine and phosphocreatine) which generate the Cre signal are widely distributed throughout the brain at a concentration of $7-11 \mathrm{mmol} / \mathrm{kg}$ wet weight. The total creatine plus phosphocreatine pool remains stable under a wide variety of abnormal metabolic and physiological conditions. Creatine and phosphocreatine are present in grey matter and white matter at about equal concentrations. For these reasons, the Cre signal was used as a normalising factor for our analysis. Despite these attributes, Cre is generally the smallest signal in the voxel spectrum. Accordingly, it is influenced to a greater extent proportionally by the random noise and artifacts which may be present in the spectrum. These factors are likely to be responsible for the apparent regional variation in Cre signal which appears in fig 1.

The MRI features of patients with NP-C have not been extensively described. ${ }^{6}{ }^{43}$ Our MRI findings suggest that cerebral and cerebellar atrophy and white matter abnormalities, although non-specific, are often present in NP-C. The significant correlation between brain atrophy and clinical score scales increases the value of MRI in evaluating patients with NP-C.

Significant correlations were found between the clinical stage and decreased NA/Cre in the frontal cortex and caudate, increased Cho/Cre in the frontal cortex, and the percentage of abnormal ROIs for both NA/Cre and Cho/Cre. The limited number of significant correlations 
found between clinical rating scale scores and metabolite ratios may be due to the few patients and the negative effect of the undetected spectroscopic voxels in several ROIs. Nevertheless, these results support the usefulness of ${ }^{1} \mathrm{H}-\mathrm{MRSI}$ in differentiating among patients with different degrees of severity of clinical abnormalities. In particular, with both the degree of abnormality of NA/Cre and Cho/ Cre and the topographical distribution of these abnormalities, ${ }^{1} \mathrm{H}-\mathrm{MRSI}$ is likely to become a sensitive tool to evaluate brain involvement in NP-C, and possibly other neurodegenerative diseases.

To our knowledge, there is only one previous ${ }^{1} \mathrm{H}-\mathrm{MRS}$ study of one patient with NP-C. ${ }^{44}$ Proton spectra, acquired from a single supraventricular volume of interest containing some grey but mostly white matter, showed normal Cho/Cre and NA/Cre and the presence of an abnormal lipid peak (at TE of $136 \mathrm{~ms}$ ). Treatment with cholesterol lowering agents caused the disappearance of the lipid peak at 13 and 19 months. Our results are not consistent with previous findings. ${ }^{43}$ We found regionally reduced NA/Cre and increased Cho/Cre but no abnormal lipid peaks in any of the ROIs of our patients. This lack of an abnormal lipid peak may be due to the different TE (272 ms) used in the present study, which is less sensitive for detecting peaks from free lipids. Indeed, at TE of $272 \mathrm{~ms}$, no lipid peak was evident. ${ }^{44}$ We suggest that these discrepancies reflect the advantages of multislice ${ }^{1} \mathrm{H}-\mathrm{MRSI}$ that permits a more detailed approach to studying diffuse CNS disorders.

The inclusion criteria used in the present study led to the exclusion of several spectroscopic voxels, and in some ROIs, no spectroscopic data could be collected at all. This was due to a combination of two limiting factors. Firstly, the size of the anatomical structure influences the number of spectroscopic voxels that could be selected, which resulted in only limited information from small but important structures (the caudate). Therefore, we were unable to explore all the structures (the hypothalamus) that may play an important part in the disease. Secondly, the quality of the spectra depends on the location of the anatomical region. The signal from some regions (the posterior fossa structures) could be affected by partial magnetic field (B0) inhomogeneities due to the proximity of sinuses and bones. The changes detected may be influenced by the number of good spectra acquired for individual ROIs. We chose to analyse only good spectra, as bad spectra were also found in normal controls, and we were thus unable to positively attribute the bad spectra to the disease. We suggest that these inclusion criteria lead to underestimating the degree of regional involvement in patients with NP-C.

Metabolite relaxation times ( $\mathrm{T} 1$ and $\mathrm{T} 2$ ) were not measured because of the prohibitively long examination times required. We used a relatively long $\mathrm{TR}(\mathrm{TR} /(\mathrm{T} 1$ of $\mathrm{NA})>1)$ and long TE $(\mathrm{TE} /(\mathrm{T} 2$ of $\mathrm{NA})<1)$ for data acquisition. The $\mathrm{T} 1$ and $\mathrm{T} 2$ relaxation time differences of ${ }^{1} \mathrm{H}$-MRSI metabolites could poten- tially cause differences in signal intensities. Therefore, we cannot exclude $\mathrm{T} 1$ or $\mathrm{T} 2$ effects on calculated ratios. Yet, to our knowledge, relaxation times of ${ }^{1} \mathrm{H}$-MRSI metabolites have not been measured in patients with NP-C. Furthermore, the regional concordance between the reduced NA signal and the neuronal loss in pathological studies suggests that the present ${ }^{1} \mathrm{H}-\mathrm{MRSI}$ findings in NP-C represent a true finding rather than the effect of a parallel pattern of changes in $\mathrm{T} 1$ or $\mathrm{T} 2$ of ${ }^{1} \mathrm{H}-\mathrm{MRSI}$ metabolites.

In conclusion, in patients with NP-C, ${ }^{1} \mathrm{H}$-MRSI showed a diffuse pattern of neuronal involvement and possibly demyelination or astrogliosis. The ${ }^{1} \mathrm{H}-\mathrm{MRSI}$ enables discrimination between patients at different stages of the disease. Moreover, ${ }^{1} \mathrm{H}-\mathrm{MRSI}$ is a useful non-invasive and objective method in the clinical evaluation of patients with NP-C, and may find application in therapeutic trials directed at the primary metabolic defect in this disease.

We thank CTW Moonen and JH Duyn for making the ${ }^{1} \mathrm{H}$-MRSI sequence available. We also appreciate the skilful editing of DG Schoenberg.

1 Pentchev PG, Vanier MT, Suzuki K, et al. Niemann-Pick disease type C: a cellular cholesterol lipidosis. In: Scriver CR, Beaudet AL, Sly WS, et al, eds. Metabolic basis of inherited disease. 7th ed. New York: McGraw-Hill, 1995:262539.

2 Vanier MT, Wenger DA, Comly ME, et al. Niemann-Pick disease group C: clinical variability and diagnosis based on defective cholesterol esterification. Clin Genet 1988;33: $331-48$

3 Sokol J, Blanchette-Mackie J, Kruth HS, et al. Type C Niemann-Pick disease. Lysosomal accumulation and defective intracellular mobilization of low density lipoprotein cholesterol. F Biol Chem 1988;263:3411-7.

4 Higgins JJ, Patterson MC, Dambrosia JM, et al. A clinical taging classification for type C Niemann-Pick disease. Neurology 1992;42:2286-90.

5 Lake BD. Lysosomal and peroxisomal disorders. In: Greenfield JD, ed. Greenfield's neuropathology. 5th ed. New York: Oxford University Press 1982:709-810.

6 Suzuki K, Parker CC, Pentchev PG, et al. Neurofibrillary tangles in Niemann-Pick disease type C. Acta Neuropathol 1995;89:227-38.

7 Barker PB, Gillard JH, van Ziil PCM, et al. Acute stroke: evaluation with serial proton MR spectroscopic imaging. Radiology 1994:192:723-32.

8 Tedeschi G, Schiffmann R, Shih H, et al. Proton magnetic resonance spectroscopic imaging in childhood ataxia with diffuse white matter hypomyelination. Neurology 1995;45: 1526-32

9 Kruse B, Barker PB, van Zijl PCM, et al. Multislice proton magnetic resonance spectroscopic imaging in X-linked adrenoleukodystrophy. Ann Neurol 1994;36:595-608.

10 Tedeschi G, Bertolino A, Massaquoi SG, et al. Proton magnetic resonance spectroscopic imaging in cerebellar degeneration. Ann Neurol 1996;39:71-8.

11 Barker PB, Lee RR, McArthur JC. AIDS dementia complex: evaluation with proton MR spectroscopic imaging. Radiology 1995;195:58-64.

12 Tedeschi G, Bertolino A, Lundbom N, et al. Cortical and subcortical chemical pathology in Alzheimer's disease as assessed by multislice proton magnetic resonance spectroscopic imaging. Neurology 1996;47:696-704.

13 Duyn JH, Gillen J, Sobering G, et al. Multisection proton Duyn JH, Gillen J, Sobering G, et al. Multisection proton
MR spectroscopic imaging of the brain. Radiology 1993; 188:277-82.

14 Hahn FJY, Rim K. Frontal ventricular dimensions on normal computed tomography. Am f Roentgenol 1976;126: $593-6$

15 Blin J, Baron JC, Dubois B, et al. Positron emission tomography study in progressive supranuclear palsy. Brain hypometabolic pattern and clinicometabolic correlations. Arch Neurol 1990;47:747-52.

16 Birken DL, Oldendorf WH. N-acetyl-L-aspartic acid: a literature review of a compound prominent in ${ }^{1} \mathrm{H}-\mathrm{NMR}$ spectroscopic studies of brain. Neurosci Biobehav Rev 1989; 13:23-31.

17 Urenjak J, Williams SR, Gadian DG, et al. Proton nuclear magnetic resonance spectroscopy unambiguously identifies different neural cell types. 7 Neurosci 1993;13:981-9.

18 Peeling J, Sutherland G. High-resolution ${ }^{1} \mathrm{H}$ NMR spectroscopy studies of extracts of human cerebral neoplasms. copy studies of extracts of human
Magn Reson Med 1992;24:123-36.

19 Gill SS, Thomas DG, Van Bruggen N, et al. Proton MR spectroscopy of intracranial tumors: in vivo and in vitro studies. F Comput Assist Tomogr 1990;14:497-504. 
20 Moffett JR, Namboodiri MA, Cangro CB, et al. Immunohistochemical localization of $\mathrm{N}$-acetylaspartate in rat brain. tochemical localization of

21 Noffett JR, Namboodiri MA, Neale JH. Enhanced carbodiimide fixation for immuno-histochemistry: application to the comparative distributions of $\mathrm{N}$-acetylaspartylglutamate and N-acetylaspartate immunoreactivities in rat brain. $f$ Histochem Cytochem 1993;41:559-70.

22 Benner RE, Munro PMG, Williams SCR, et al. The proton NMR spectrum in acute EAE: the significance of the change in the Cho:Cr ratio. Magn Reson Med 1993;29:73745.

23 Rango M, Spagnoli D, Tomei G, et al. Central nervous system trans-synaptic effects of acute axonal injury: a ${ }^{1} \mathrm{H}$ magnetic resonance spectroscopy study. Magn Reson Med 1995;33:595-600.

24 Vion-Dury J, Nicoli F, Salvan AM, et al. Reversal of brain metabolic alterations with zido-vudine detected by proton localized magnetic resonance spectroscopy. Lancet 1995; 345:60-1.

25 De Stefano N, Matthews PM, Antel JP, et al. Chemical pathology of acute demyelinating lesions and its correlapathology of acute demyelinating lesions and
tions with disability. Ann Neurol 1995;38:901-9.

26 Miller BL. A review of chemical issues in ${ }^{1} \mathrm{H}$ NMR spectroscopy: N-acetylaspartate, creatine and choline. NMR Biomed 1991;4:47-52.

27 Miller BL, Chang L, Booth R, et al. In vivo ${ }^{1} \mathrm{H}$ MRS choline: correlation with in vitro chemistry/histology. Life Sci 1996, 58:1929-35.

28 Arnold DL, Matthews PM, Francis G, et al. Proton magnetic resonance spectroscopy of human brain in vivo in the evaluation of multiple sclerosis: assessment of the load of disease. Magn Reson Med 1990;14:154-9.

29 Larsson HBW, Christiansen P, Jensen M, et al. Localized in vivo proton spectroscopy in the brain of patients with multiple sclerosis. Magn Reson Med 1991;22:23-31.

30 Arnold DL, Matthews PM, Francis G, et al. Proton magnetic resonance spectroscopic imaging for metabolic magnetic resonance spectroscopic imaging for metabolic characterization

31 Tzika AA, Ball WS, Vigneron DB, et al. Clinical proton spectroscopy of neurodegenerative disease in childhood. spectroscopy of neurodegenerative disease
AfNR Am $\mathcal{~}$ Neuroradiol 1993;14:1267-81.

32 Bruhn H, Kruse B, Korenke GC, et al. Proton NMR spec- troscopy of cerebral metabolic alterations in infantile paroxysmal disorders. F Comput Assist Tomogr 1992;16: paroxys $335-44$.

33 Tzika AA, Ball WS, Vigneron DB, et al. Childhood adrenoleukodystrophy: assessment by proton MR spectroscopy. Radiology 1993;189:467-80.

34 Detre JA, Wang Z, Bogdan AR, et al. Regional variation in brain lactate in Leigh syndrome by localized ${ }^{1} \mathrm{H}$ magnetic resonance spectroscopy. Ann Neurol 1991;29:218-21.

35 Krageloh-Mann I, Grodd W, Nieman G, et al. Assessment and therapy monitoring of Leigh disease by MRI and proton spectroscopy. Pediatr Neurol 1992;8:60-4.

36 Grodd W, Krageloh-Mann I, Klose U, et al. Metabolic and destructive brain disorders in children: findings with localized proton MR spectroscopy. Radiology 1991;181:173-81.

37 Grodd W, Krageloh-Mann I, Petersen D, et al. In vivo assessment of $\mathrm{N}$-acetylaspartate in brain in spongy degeneration (Canavan's disease) by proton spectroscopy. Lancet 1990;336:437-8.

38 Marks HG, Caro AP, Wang Z, et al. Use of computed tomography, magnetic resonance imaging, and localized ${ }^{1} \mathrm{H}$ ography, magnetic resonance imaging, and localized ' $\mathrm{H}$
magnetic resonance spectroscopy in Canavan's disease: a magnetic resonance spectroscopy in Can

39 van der Knapp MS, van der Grond J, Luyten PR, et al. ${ }^{1} \mathrm{H}$ and ${ }^{31} \mathrm{P}$ magnetic resonance spectroscopy of the brain in degenerative cerebral disorders. Ann Neurol 1992;31:20211 .

40 Negendank W. Studies of human tumors by MRS: a review. NMR Biomed 1992;5:303-24.

41 Fulham MJ, Bizzi A, Dietz MJ, et al. Mapping of brain tumor metabolites with proton MR spectroscopic imaging: clinical relevance. Radiology 1992;185:675-86.

42 Petroff OA, Pleban LA, Spencer DD. Symbiosis between in vivo and in vitro NMR spectroscopy: the creatine, $\mathrm{N}$-acetylaspartate, glutamate, and GABA content of the epileptic human brain. Magn Reson Imaging 1995;13:1197211.

43 Palmieri S, Battisti C, Federico A, et al. Hypoplasia of the corpus callosum in Niemann-Pick type C disease. Neurora194;36:20-2.

44 Sylvain M, Arnold DL, Scriver CR, et al. Magnetic resonance spectroscopy in Niemann-Pick disease type C: correlation with diagnosis and clinical response to cholestyramine and lovastatin. Pediatr Neurol 1994;10:228-32. 